\title{
On Relations among Fourier Coefficients and Sum-functions*
}

\author{
D. S. Yu and S. P. Zhou
}

\begin{abstract}
We generalize five theorems of Leindler on the relations among Fourier coefficients and sum-functions under the more general $N B V$ condition.

Keywords. Fourier coefficients; sum-functions; $N B V$ condition.
\end{abstract}

2000 Mathematics Subject Classification: 42A32, 42A16.

\section{$\S 1$. Introduction}

Let $f(x)$ be a $p$ power integrable function of period $2 \pi$, in symbol, $f \in L^{p}, p \geq 1$. Define

$$
\omega_{p}(f, h):=\sup _{|t| \leq h}\|f(x+t)-f(x)\|_{p}
$$

and

$$
\omega_{p}^{*}(f, h):=\sup _{0<t \leq h}\|f(x+t)+f(x-t)-2 f(x)\|_{p},
$$

where $\|\cdot\|_{p}$ denotes the usual $L^{p}$ norm.

Denote by $E_{n}^{(p)}(f)$ the best approximation of order $n$ of $f$ in $L^{p}$. The Lipschitz class $\Lambda_{p}$ and the Zygmund class $\Lambda_{p}^{*}$ are defined by

$$
\Lambda_{p}:=\left\{f \in L^{p}: \omega_{p}(f, h)=O(h)\right\}
$$

\footnotetext{
*Supported in part by Natural Science Foundation of China under grant number 10471130.
} 
and

$$
\Lambda_{p}^{*}:=\left\{f \in L^{p}: \omega_{p}^{*}(f, h)=O(h)\right\}
$$

respectively.

Leindler [5] introduced a class of sequences, a natural extension of monotone decreasing sequences, named as $R B V S$. Namely, a sequence $\mathbf{C}:=\left\{c_{n}\right\}$ of nonegative numbers tending to zero is called of "rest bounded variation", written as $\mathbf{C} \in R B V S$, if it satisfies

$$
\sum_{n=m}^{\infty}\left|\Delta c_{n}\right| \leq K(\mathbf{C}) c_{m}
$$

for all $m=1,2, \cdots$, where $K(\mathbf{C})$ is a constant only depending upon $\mathbf{C}$ and $\Delta c_{n}=$ $c_{n}-c_{n+1}, n=1,2, \cdots$.

Leindler [6] pointed out that $R B V S$ and the well known quasi-monotone sequences $(C Q M S)$ are not comparable. Very recently, Le and Zhou [2] defined a new condition named as $G B V$ condition to include both the $R B V$ and quasi-monotone conditions. In the special real case, the $G B V$ condition can be stated as follows: Let $\mathbf{A}:=\left\{a_{n}\right\}$ be a sequence of nonegative numbers, if

$$
\sum_{m=n}^{2 n}\left|\Delta a_{n}\right| \leq K(\mathbf{A}) a_{n}
$$

for all $n=1,2, \cdots$, then we say $\mathbf{A}$ satisfies the $G B V$ condition, briefly, write $\mathbf{A} \in$ $G B V S$. Many important classic results in Fourier analysis could be generalized by replacing the monotonicity of coefficients by $R B V$ or $G B V$ condition. For example, readers could refer to [11] for more information. Recently, we [8] further introduced a new kind of sequences named as $N B V S$. In the real case, the $N B V S$ can be defined as follows. Let $\mathbf{A}:=\left\{a_{n}\right\}$ be a sequence of nonegative numbers, if

$$
\sum_{m=n}^{2 n}\left|\Delta a_{n}\right| \leq K(\mathbf{A})\left(a_{n}+a_{2 n}\right)
$$

for all $n=1,2, \cdots$, then we say A satisfies the $N B V$ condition, briefly, write $\mathbf{A} \in$ $N B V S$. As we know, the following embedding relations

$$
R B V S \cup C Q M S \subset G B V S \subset N B V S
$$


holds. Furthermore, as we mentioned in [8], NBVS can be regarded as a "two-sided" monotonicity condition.

For convenience, through out the paper, we use $K$ to indicate a positive constant which may depend upon $p$ and $\mathbf{A}$, its value may be different even in the same line.

\section{§2. Main Results}

In this paper, we will establish the following results on the relations among Fourier coefficients and the sum-functions. All of them were proved for $R B V S$ by Leindler [7], and Theorem 1 was proved for GBVS by Zhou and Le [10].

Theorem 1. Let $\mathbf{A} \in N B V S$ be such for a fixed $p, 1<p<\infty$, that

$$
\sum_{n=1}^{\infty} n^{p-2} a_{n}^{p}<\infty
$$

If $f$ is the sum of either of the series

$$
\sum_{n=1}^{\infty} a_{n} \cos n x \quad \text { or } \quad \sum_{n=1}^{\infty} a_{n} \sin n x
$$

then

$$
\omega_{p}\left(f, n^{-1}\right) \leq K_{1} n^{-1}\left\{\sum_{\nu=1}^{n-1} \nu^{2 p-2} a_{\nu}^{p}\right\}^{1 / p}+K_{2}\left\{\sum_{\nu=n}^{\infty} \nu^{p-2} a_{\nu}^{p}\right\}^{1 / p} .
$$

Theorem 2. Let $1<p<\infty, 1 \leq r<\infty$ and $\lambda(x), x \geq 1$, be a positive monotone function with $K_{1} \lambda\left(2^{n}\right) \leq \lambda\left(2^{n+1}\right) \leq K_{2} \lambda\left(2^{n}\right)$, where $K_{1}>0$. Write $\mathbf{A}=$ $\left\{a_{n}\right\} \in N B V S, f(x)=\sum_{k=1}^{\infty} a_{k} \cos k x \in L^{p}$. Then

$$
\begin{aligned}
& \sum_{n=1}^{\infty} \lambda(n) a_{n}^{r} \leq K I(f, \lambda, r, p):= \\
& K \int_{0}^{1} \lambda\left(\frac{1}{t}\right) t^{r-2-\frac{r}{p}}\left(\int_{0}^{\pi}|f(x+t)+f(x-t)-2 f(x)|^{p} d x\right)^{r / p} d t .
\end{aligned}
$$

If $\sum_{n=1}^{\infty}\left|\Delta a_{n}\right|<\infty$ and $\lambda(x)$ satisfies the additional conditions

$$
\sum_{n=1}^{m} \lambda(n) n^{\frac{r}{p}-r} \leq K \lambda(m) m^{\frac{r}{p}-r+1}
$$


and

$$
\sum_{n=m}^{\infty} \lambda(n) n^{r\left(\frac{1}{p}-3\right)} \leq K \lambda(m) m^{1+r\left(\frac{1}{p}-3\right)}
$$

then

$$
I(f, \lambda, r, p) \leq K \sum_{n=1}^{\infty} \lambda(n) a_{n}^{r} .
$$

Theorem 3. Let $1<p<r$ and $\left\{\varphi_{n}\right\}$ be a nonnegative nondecreasing sequence satisfying $\varphi_{n^{2}} \leq K \varphi_{n}$ for all $n$. Define

$$
\Phi(x):=\sum_{n=1}^{x} n^{\frac{r}{p}-2} \varphi_{n}
$$

where $\varphi(x):=\varphi_{n}$ if $x \in(n-1, n)$. Write $\mathbf{A}=\left\{a_{n}\right\} \in N B V S, f(x)=\sum_{k=1}^{\infty} a_{k} \cos k x \in$ $L^{p}$. Then the statements

$$
\begin{gathered}
\sum_{n=1}^{\infty} \varphi_{n} n^{r-2} a_{n}^{r}<\infty \\
\sum_{n=1}^{\infty} \varphi_{n} n^{r s+\frac{r}{p}-2}\left(\sum_{k=1}^{n} k^{(s+1) p-2} a_{k}^{p}\right)^{r / p}<\infty \text { for any } s>\frac{1}{p}-\frac{1}{r} \\
\sum_{n=1}^{\infty} \varphi_{n} n^{\frac{r}{p}-2}\left(\sum_{k=n}^{\infty} k^{p-2} a_{k}^{p}\right)^{r / p}<\infty \\
\sum_{n=1}^{\infty} \varphi_{n} n^{\frac{r}{p}-2}\left(\omega_{p}\left(f, \frac{1}{n}\right)\right)^{r}<\infty \\
\sum_{n=1}^{\infty} \varphi_{n} n^{\frac{r}{p}-2}\left(E_{n}^{(p)}(f)\right)^{r}<\infty \\
\int_{0}^{\pi}|f(x)|^{r-\frac{r}{p}+1} \Phi(|f(x)|) d x<\infty \\
\int_{0}^{\pi}|f(x)|^{r} \varphi(|f(x)|) d x<\infty \\
\int_{0}^{\pi}|f(x)|^{r} \varphi\left(\frac{1}{x}\right) d x<\infty
\end{gathered}
$$

and

$$
\int_{0}^{\pi} \varphi\left(\frac{1}{t}\right) t^{-\frac{r}{p}}\left(\int_{0}^{\pi}|f(x+t)+f(x-t)-2 f(x)|^{p} d x\right)^{r / p}<\infty
$$

are equivalent. 
Theorem 4. If $1<p<\infty$ and $\mathbf{A} \in N B V S$, then

$$
\sum_{n=1}^{\infty} n^{2 p-2} a_{n}^{p}<\infty
$$

is a necessary and sufficient condition that a sum-function of either of the series (2.2)

(i) belongs to $\Lambda_{p}$, or

(ii) is equivalent to an absolutely continuous function whose derivative belongs to $L^{p}$.

Theorem 5. If $f \in L^{p}, 1<p<\infty, \mathbf{A} \in N B V S$ and $f$ is a sum-function of either the series (2.2), then $f \in \Lambda_{p}^{*}$ implies that

$$
\omega_{p}(f, h) \leq K h|\log h|^{1 / p} .
$$

The proofs of the above results will be proceeded as in a way as those of Leindler [7], only necessary modifications will be noted.

\section{§3. Lemmas}

Lemma 1. Let $1<p<\infty, 1 \leq r<\infty$ and $\lambda(x), x \geq 1$, be a positive monotone function with $K_{1} \lambda\left(2^{n}\right) \leq \lambda\left(2^{n+1}\right) \leq K_{2} \lambda\left(2^{n}\right)$, where $K_{1}>0$. Let $a_{n} \geq 0$, $n=1,2, \cdots$, and write $f(x)=\sum_{k=1}^{\infty} a_{k} \cos k x \in L^{p}$. Then

$$
\sum_{n=1}^{\infty} \lambda(n) n^{-r}\left(\sum_{k=[n / 2]}^{2 n} a_{k}\right)^{r} \leq K I(f, \lambda, r, p) .
$$

If $\sum_{n=1}^{\infty}\left|\Delta a_{n}\right|<\infty$ and $\lambda(x)$ satisfies (2.5) and (2.6), then

$$
I(f, \lambda, r, p) \leq K \sum_{n=1}^{\infty} \lambda(n)\left(\sum_{k=n}^{\infty}\left|\Delta a_{k}\right|\right)^{r} .
$$

Proof. The second result can be found directly in [4], while the first can be proved by the same argument as of [4].

Lemma 2([9]). Let $1<p<\infty,\left\{a_{n}\right\} \in N B V S$, and $f$ be the sum of either of the series (2.2), then $f \in L^{p}$ if and only if (2.1) holds. 
Lemma 3([3]). Let $\alpha_{n} \geq 0$ and $\lambda_{n} \geq 0$ be given, $\nu_{1}<\cdots<\nu_{n}<\cdots$ denote the indices for which $\lambda_{\nu_{n}}>0$, and $N$ denote the number of positive terms of the sequence $\lambda_{n}$, provides this number is finite, or in the contrary case set $N=\infty$. Set $\nu_{0}=0$ and if $N<\infty$ then $\nu_{N+1}=\infty$. We have the following inequalities:

$$
\begin{gathered}
\sum_{n=1}^{\infty} \lambda_{n}\left(\sum_{k=1}^{n} \alpha_{k}\right)^{p} \leq p^{p} \sum_{n=1}^{\infty} \lambda_{\nu_{n}}^{1-p}\left(\sum_{k=\nu_{n}}^{\infty} \lambda_{k}\right)^{p}\left(\sum_{k=\nu_{n-1}+1}^{\nu_{n}} \alpha_{k}\right)^{p}, \\
\sum_{n=1}^{\infty} \lambda_{n}\left(\sum_{k=n}^{\infty} \alpha_{k}\right)^{p} \leq p^{p} \sum_{n=1}^{N} \lambda_{\nu_{n}}^{1-p}\left(\sum_{k=1}^{\nu_{n}} \lambda_{k}\right)^{p}\left(\sum_{k=\nu_{n}}^{\nu_{n+1}-1} \alpha_{k}\right)^{p} .
\end{gathered}
$$

Lemma 4([9]). If $\mathbf{A}:=\left\{a_{n}\right\} \in N B V S$, then for all $n \geq 1$, it holds that

$$
I:=\sum_{k=n}^{\infty}\left|\Delta a_{k}\right| \leq C(\mathbf{A})\left(a_{n}+a_{2 n}+a_{4 n}+\sum_{k=n}^{\infty} \frac{a_{k}}{k}\right) .
$$

Lemma 5. If $\mathbf{A}:=\left\{a_{n}\right\} \in N B V S$, then

$$
n^{-p} \sum_{m=1}^{n-1} m^{-2}\left(\sum_{\nu=1}^{m} \nu^{2}\left|\Delta a_{\nu}\right|\right)^{p} \leq K\left(n^{-p} \sum_{\nu=1}^{n-1} \nu^{2 p-2} a_{\nu}^{p}+\sum_{\nu=n}^{\infty} \nu^{p-2} a_{\nu}^{p}\right) .
$$

Proof. Write

$$
N(x)=: \frac{\log x}{\log 2}, \quad x>0 .
$$

Since $m / 4 \leq 2^{[N(m / 2)]} \leq m / 2$ for $m \geq 2$, then

$$
\sum_{\nu=1}^{m} \nu^{2}\left|\Delta a_{\nu}\right| \leq \sum_{k=1}^{[N(m / 2)]} \sum_{\nu=2^{k-1}}^{2^{k}} \nu^{2}\left|\Delta a_{\nu}\right|+\sum_{k=[m / 4]+1}^{m} \nu^{2}\left|\Delta a_{\nu}\right|=: J_{1}+J_{2} .
$$

By the definition of $N B V S$, we get

$$
a_{2^{k}} \leq \sum_{i=s}^{2^{k}}\left|\Delta a_{i}\right|+a_{s} \leq \sum_{i=s}^{2 s}\left|\Delta a_{i}\right|+a_{s} \leq K\left(a_{s}+a_{2 s}\right),
$$

and

$$
a_{2^{k-1}} \leq \sum_{i=2^{k-1}}^{s-1}\left|\Delta a_{i}\right|+a_{s} \leq K\left(a_{[s / 2]}+a_{s}\right)
$$

for all $2^{k-1} \leq s \leq 2^{k}$, and hence deduce that

$$
\begin{aligned}
J_{1} & \leq \sum_{k=1}^{[N(m / 2)]} 2^{2 k} \sum_{\nu=2^{k-1}}^{2^{k}}\left|\Delta a_{\nu}\right| \leq K \sum_{k=1}^{[N(m / 2)]} 2^{2 k}\left(a_{2^{k-1}}+a_{2^{k}}\right) \\
& \leq K \sum_{k=1}^{[N(m / 2)]} 2^{k} \sum_{s=2^{k-1}}^{2^{k}}\left(a_{[s / 2]}+a_{s}+a_{2 s}\right) \leq K \sum_{k=1}^{m} k a_{k} .
\end{aligned}
$$


For any $[m / 4]+1 \leq s \leq 2[m / 4]+2$, if $s$ is an even number, it follows from the definition of $N B V S$ that

$$
\begin{aligned}
\left|a_{[m / 4]+1}\right| & \leq \sum_{k=[m / 4]+1}^{s-1}\left|\Delta a_{k}\right|+a_{s} \leq \sum_{k=[s / 2]}^{s}\left|\Delta a_{k}\right|+a_{s} \\
& \leq K\left(a_{s}+a_{[s / 2]}\right) .
\end{aligned}
$$

If $s$ is an odd number, then

$$
\begin{aligned}
\left|a_{[m / 4]+1}\right| & \leq \sum_{k=[m / 4]+1}^{s-1}\left|\Delta a_{k}\right|+a_{s} \leq \sum_{k=[s / 2]}^{s-1}\left|\Delta a_{k}\right|+a_{s} \\
& \leq K\left(a_{s}+a_{s-1}+a_{[s / 2]}\right) .
\end{aligned}
$$

Therefore, in any case,

$$
m^{2} a_{[m / 4]+1} \leq K \sum_{s=[m / 4]+1}^{2[m / 4]+2} s\left(a_{s}+a_{s-1}+a_{[s / 2]}\right) \leq K \sum_{k=1}^{m} k a_{k} .
$$

A similar discussion leads to

$$
m^{2}\left|a_{2[m / 4]+2}\right| \leq K \sum_{k=[m / 4]+2}^{4[m / 4]} s\left(a_{s}+a_{s-1}+a_{[s / 2]}\right) \leq K \sum_{k=1}^{m} k a_{k},
$$

and

$$
m^{2}\left|a_{[m / 2]}\right| \leq K \sum_{k=[m / 2]}^{m} s\left(a_{s}+a_{s-1}+a_{[s / 2]}\right) \leq K \sum_{k=1}^{m} k a_{k} .
$$

Set

$$
m^{*}=: \begin{cases}m, & m \text { is even, } \\ m-1, & m \text { is odd }\end{cases}
$$

By (3.5)-(3.7), we deduce that

$$
\begin{aligned}
J_{2} & \leq \sum_{\nu=[m / 4]+1}^{2[m / 4]+2} \nu^{2}\left|\Delta a_{\nu}\right|+\sum_{\nu=[m / 2]}^{m^{*}} \nu^{2}\left|\Delta a_{\nu}\right|+m^{2}\left(a_{m}+a_{m+1}\right) \\
& \leq K m^{2}\left(a_{[m / 4]+1}+a_{2[m / 4]+2}+a_{m^{*} / 2}+a_{m^{*}}+a_{m}+a_{m+1}\right) \\
& \leq K m^{2}\left(a_{[m / 4]+1}+a_{2[m / 4]+2}+a_{[m / 2]}+a_{m-1}+a_{m}+a_{m+1}\right) \\
& \leq K \sum_{k=1}^{m} k a_{k}+K m^{2}\left(a_{m-1}+a_{m}+a_{m+1}\right) .
\end{aligned}
$$


Combining all the estimates for $J_{1}$ and $J_{2}$ with the fact (see [7])

$$
n^{-p} \sum_{m=1}^{n-1} m^{-2}\left(\sum_{\nu=1}^{m} \nu a_{\nu}\right)^{p} \leq K n^{-p} \sum_{\nu=1}^{n-1} \nu^{2 p-2} a_{\nu}^{p}
$$

we see that

$$
\begin{aligned}
n^{-p} \sum_{m=1}^{n-1} m^{-2}\left(\sum_{\nu=1}^{m} \nu^{2}\left|\Delta a_{\nu}\right|\right)^{p} \leq K n^{-p} \sum_{m=1}^{n-1} m^{-2}\left(\sum_{k=1}^{m} k a_{k}\right)^{p} \\
+K n^{-p} \sum_{m=1}^{n-1} m^{2 p-2}\left(a_{m-1}^{p}+a_{m}^{p}+a_{m+1}^{p}\right) \\
\leq K n^{-p} \sum_{\nu=1}^{n-1} \nu^{2 p-2} a_{\nu}^{p}+K n^{-p} \sum_{m=1}^{n-1} m^{2 p-2} a_{m+1}^{p} \\
\leq K n^{-p} \sum_{\nu=1}^{n-1} \nu^{2 p-2} a_{\nu}^{p}+K n^{p-2} a_{n}^{p} \\
\leq K n^{-p} \sum_{\nu=1}^{n-1} \nu^{2 p-2} a_{\nu}^{p}+K \sum_{\nu=n}^{\infty} \nu^{p-2} a_{\nu}^{p}
\end{aligned}
$$

Lemma 6. If $\mathbf{A}:=\left\{a_{n}\right\} \in N B V S$, then

$$
n^{-p} \sum_{m=1}^{n-1} m^{p-2}\left(\sum_{\nu=m+1}^{n} \nu\left|\Delta a_{\nu}\right|\right)^{p} \leq K\left(n^{-p} \sum_{\nu=1}^{n-1} \nu^{2 p-2} a_{\nu}^{p}+\sum_{\nu=n}^{\infty} \nu^{p-2} a_{\nu}^{p}\right) .
$$

Proof. First assume that $n \geq 8(m+1)$. By noting that $m+1 \leq 2^{[N(m+1)]+1} \leq$ $2(m+1)$ and $n / 4 \leq 2^{[N(n / 2)]} \leq n / 2$, we can split $\sum_{k=m+1}^{n} \nu\left|\Delta a_{\nu}\right|$ into

$$
\begin{gathered}
\sum_{\nu=m+1}^{n} \nu\left|\Delta a_{\nu}\right| \leq \sum_{k=[N(m+1)]+3}^{N(n / 2)} \sum_{\nu=2^{k-1}}^{2^{k}} \nu\left|\Delta a_{\nu}\right|+\sum_{\nu=[n / 4]+1}^{n} \nu\left|\Delta a_{\nu}\right|+\sum_{\nu=m+1}^{2^{[N(m+1)]+3}-1} \nu\left|\Delta a_{\nu}\right| \\
=: H_{1}+H_{2}+H_{3} .
\end{gathered}
$$

Similar to what we have done for $J_{1}$ in the proof of Lemma 5 , we get

$$
H_{1} \leq K \sum_{\nu=m+1}^{n} a_{\nu}
$$


Since $n \geq 8(m+1)$, then setting

$$
N^{*}=:\left[\frac{1}{2}([n / 4]+1)\right] \geq m+1,
$$

again similar to $J_{2}$, we have

$$
\begin{aligned}
& H_{2} \leq K \sum_{k=N^{*}}^{n} a_{k}+K n\left(a_{n-1}+a_{n}+a_{n+1}\right) \\
& \leq K \sum_{k=m+1}^{n} a_{k}+K n\left(a_{n-1}+a_{n}+a_{n+1}\right) .
\end{aligned}
$$

At the same time, it is evident that

$$
H_{3} \leq \sum_{\nu=m+1}^{8(m+1)} \nu\left|\Delta a_{\nu}\right| \leq K m\left(a_{m+1}+a_{2(m+1)}+a_{4(m+1)}+a_{8(m+1)}\right) .
$$

In case $n<8(m+1)$, then it clearly holds that

$$
\sum_{\nu=m+1}^{n} \nu\left|\Delta a_{\nu}\right| \leq K m\left(a_{m+1}+a_{2(m+1)}+a_{4(m+1)}+a_{8(m+1)}\right) .
$$

Altogether, all the above estimates lead to that

$$
\begin{gathered}
n^{-p} \sum_{m=1}^{n-1} m^{p-2}\left(\sum_{\nu=m+1}^{n} \nu\left|\Delta a_{\nu}\right|\right)^{p} \leq K n^{-p} \sum_{m=1}^{n-1} m^{p-2}\left(\sum_{\nu=m+1}^{n} a_{\nu}\right)^{p} \\
+K n^{p-1}\left(a_{n-1}^{p}+a_{n}^{p}+a_{n+1}^{p}\right)+K n^{-p} \sum_{m=1}^{n-1} m^{2 p-2} \sum_{j=1}^{8} a_{j(m+1)}^{p} .
\end{gathered}
$$

Obviously,

$$
a_{n} \leq \sum_{i=k}^{n-1}\left|\Delta a_{i}\right|+a_{k} \leq K\left(a_{k}+a_{2 k}\right)
$$

for $[n / 2]+1 \leq k \leq n$, which implies that

$$
a_{n} \leq K n^{-1} \sum_{k=[n / 2]+1}^{n-1}\left(a_{k}+a_{2 k}\right) \leq K n^{-1} \sum_{k=[n / 2]+1}^{2 n-2} a_{k}
$$

so that applying Hölder's inequality yields that

$$
n^{p-1} a_{n}^{p} \leq K n^{-1}\left(\sum_{k=[n / 2]+1}^{2 n-2} a_{k}\right)^{p} \leq K n^{p-2} \sum_{k=[n / 2]+1}^{2 n-2} a_{k}^{p}
$$


$\leq K\left(n^{-p} \sum_{k=[n / 2]+1}^{n-1} k^{2 p-2} a_{k}^{p}+\sum_{k=n}^{2 n-2} k^{p-2} a_{k}^{p}\right) \leq K\left(n^{-p} \sum_{k=1}^{n-1} k^{2 p-2} a_{k}^{p}+\sum_{k=n}^{\infty} k^{p-2} a_{k}^{p}\right)$.

Similarly,

$$
\begin{aligned}
& n^{p-1} a_{n-1}^{p} \leq K\left(n^{-p} \sum_{k=1}^{n-1} k^{2 p-2} a_{k}^{p}+\sum_{k=n}^{\infty} k^{p-2} a_{k}^{p}\right) \\
& n^{p-1} a_{n+1}^{p} \leq K\left(n^{-p} \sum_{k=1}^{n-1} k^{2 p-2} a_{k}^{p}+\sum_{k=n}^{\infty} k^{p-2} a_{k}^{p}\right) .
\end{aligned}
$$

Since (see [7])

$$
n^{-p} \sum_{m=1}^{n-1} m^{p-2}\left(\sum_{\nu=m+1}^{n} a_{\nu}\right)^{p} \leq K n^{-p} \sum_{\nu=1}^{n-1} \nu^{2 p-2} a_{\nu}^{p}
$$

with the estimates (3.9)-(3.11), Lemma 6 will be completed if we can verify that

$$
\Delta=: n^{-p} \sum_{m=1}^{n-1} m^{2 p-2} \sum_{j=1}^{8} a_{j(m+1)}^{p} \leq K n^{-p} \sum_{\nu=1}^{n-1} \nu^{2 p-2} a_{\nu}^{p}+\sum_{\nu=n}^{\infty} \nu^{p-2} a_{\nu}^{p} .
$$

Indeed, we prove (3.12) by the following way:

$$
\begin{aligned}
\Delta & =n^{-p} \sum_{j=1}^{8} \sum_{\nu=1}^{[n / j]-1} \nu^{2 p-2} a_{j(\nu+1)}^{p}+n^{-p} \sum_{j=1}^{8} \sum_{\nu=[n / j]}^{n-1} \nu^{2 p-2} a_{j(\nu+1)}^{p} \\
& \leq K\left(n^{-p} \sum_{\nu=1}^{n-1} \nu^{2 p-2} a_{\nu}^{p}+n^{p-2} a_{n}^{p}+\sum_{j=1}^{8} \sum_{\nu=n}^{j n} \nu^{p-2} a_{\nu}^{p}\right) \\
& \leq K\left(n^{-p} \sum_{\nu=1}^{n-1} \nu^{2 p-2} a_{\nu}^{p}+\sum_{j=1}^{8} \sum_{\nu=n}^{j n} \nu^{p-2} a_{\nu}^{p}\right) \\
& \leq K\left(n^{-p} \sum_{\nu=1}^{n-1} \nu^{2 p-2} a_{\nu}^{p}+\sum_{\nu=n}^{\infty} \nu^{p-2} a_{\nu}^{p}\right)
\end{aligned}
$$

\section{§4. Proofs}

Proof of Theorem 1. By Lemma 2, we know that the condition (2.1) is both necessary and sufficient for $f \in L^{p}, p>1$. We only need to treat the cosine series case, the other could be done similarly. Assume that $h=\pi / 2 n$. By the symmetry of $f$, it is clear that

$$
\begin{gathered}
\omega_{p}(f, h) \leq K \sup _{0<t \leq h}\left(\left\{\int_{0}^{\pi / n}|f(x \pm t)-f(x)|^{p} d x\right\}^{1 / p}\right. \\
\left.+\left\{\int_{\pi / n}^{\pi}|f(x \pm t)-f(x)|^{p} d x\right\}^{1 / p}\right):=K \sup _{0<t \leq h}\left(I_{1}+I_{2}\right) .
\end{gathered}
$$


As the way done by Leindler [7], we have

$$
\begin{aligned}
\frac{1}{2} I_{1} \leq t\left\{\int_{0}^{\pi / n}\left(\sum_{\nu=1}^{n-1} \nu a_{\nu}\right)^{p} d x\right\}^{1 / p} & +K\left\{\sum_{m=n}^{\infty} \int_{3 \pi / 2(m+1)}^{3 \pi / 2 m}\left|\sum_{\nu=n}^{\infty} a_{\nu} \cos \nu x\right|^{p} d x\right\}^{1 / p} \\
= & : I_{11}+I_{12} .
\end{aligned}
$$

Applying Hölder's inequality leads to

$$
I_{11} \leq K n^{-1}\left\{\sum_{\nu=1}^{n-1} \nu^{2 p-2} a_{\nu}^{p}\right\}^{1 / p} .
$$

By Abel's transformation and Lemma 4, we have

$$
\begin{aligned}
\left|\sum_{\nu=n}^{\infty} a_{\nu} \cos \nu x\right| & \leq \sum_{\nu=n}^{m} a_{\nu}+(m+1) \sum_{\nu=m+1}^{\infty}\left|\Delta a_{\nu}\right| \\
& \leq K\left(\sum_{\nu=n}^{m} a_{\nu}+m\left(a_{m}+a_{2 m}+a_{4 m}\right)+m \sum_{\nu=m+1}^{\infty} \frac{a_{\nu}}{\nu}\right) .
\end{aligned}
$$

Setting $\lambda_{m}=m^{-2}$ and $\alpha_{m}=0$ for $m<n$ and $\alpha_{m}=a_{m}$ for $m \geq n$, we get

$$
\begin{aligned}
\sum_{m=n}^{\infty} m^{-2}\left(\sum_{\nu=n}^{m} \alpha_{\nu}\right)^{p} & =\sum_{m=1}^{\infty} m^{-2}\left(\sum_{\nu=1}^{m} \alpha_{\nu}\right)^{p} \\
& \leq K \sum_{m=1}^{\infty} m^{p-2} \alpha_{m}^{p}=K \sum_{m=n}^{\infty} m^{p-2} a_{m}^{p}
\end{aligned}
$$

by (3.3). Again setting

$$
\begin{gathered}
\nu_{1}=n, \nu_{2}=n+1, \cdots, \nu_{j}=n+j, \cdots, \\
\lambda_{1}=\lambda_{2}=\cdots=\lambda_{\nu_{1}-1}=0, \lambda_{\nu_{j}}=\nu_{j}^{p-2}, j=1,2, \cdots,
\end{gathered}
$$

with (3.4), we get

$$
\begin{aligned}
\sum_{m=n}^{\infty} m^{p-2}\left(\sum_{\nu=m}^{\infty} \frac{a_{\nu}}{\nu}\right)^{p} & =\sum_{j=1}^{\infty} \lambda_{j}\left(\sum_{k=j}^{\infty} \frac{a_{k}}{k}\right)^{p} \\
& \leq p^{p} \sum_{j=1}^{\infty} \lambda_{\nu_{j}}^{1-p}\left(\sum_{k=1}^{\nu_{j}} \lambda_{k}\right)^{p}\left(\sum_{k=\nu_{j}}^{\nu_{j+1}-1} \frac{a_{k}}{k}\right)^{p} \\
& =p^{p} \sum_{m=n}^{\infty} m^{(p-2)(1-p)}\left(\sum_{k=n}^{m} k^{p-2}\right)^{p}\left(\frac{a_{m}}{m}\right)^{p} \\
& \leq p^{p} \sum_{m=n}^{\infty} m^{p-2} a_{m}^{p} .
\end{aligned}
$$


Therefore

$$
\begin{aligned}
I_{12}^{p} & \leq K \sum_{m=n}^{\infty} m^{-2}\left(\sum_{\nu=n}^{m} a_{\nu}\right)^{p}+K \sum_{m=n}^{\infty} m^{p-2} a_{m}^{p}+K \sum_{m=n}^{\infty} m^{p-2}\left(\sum_{\nu=m}^{\infty} \frac{a_{\nu}}{\nu}\right)^{p} \\
& \leq K \sum_{m=n}^{\infty} m^{p-2} a_{m}^{p} .
\end{aligned}
$$

Let $D_{\nu}(x)$ be the Dirichlet Kernel. Following the way of Leindler [7], we see that

$$
\begin{gathered}
I_{2} \leq\left\{\int_{\pi / n}^{\pi}\left|\sum_{\nu=1}^{n} \Delta a_{\nu}\left[D_{\nu}(x \pm t)-D_{\nu}(x)\right]\right|^{p} d x\right\}^{1 / p} \\
+\left\{\int_{\pi / n}^{\pi}\left|\sum_{\nu=n+1}^{\infty} \Delta a_{\nu}\left[D_{\nu}(x \pm t)-D_{\nu}(x)\right]\right|^{p} d x\right\}^{1 / p}:=I_{21}+I_{22},
\end{gathered}
$$

and

$$
\begin{array}{r}
I_{21}^{p} \leq K \sum_{m=1}^{n-1} \int_{\pi /(m+1)}^{\pi / m} \sum_{\nu=1}^{n}\left|\Delta a_{\nu}\left[D_{\nu}(x \pm t)-D_{\nu}(x)\right]\right|^{p} d x \\
\leq K t^{p}\left\{\sum_{m=1}^{n-1} m^{-2}\left(\sum_{\nu=1}^{m} \nu^{2}\left|\Delta a_{\nu}\right|\right)^{p}+\sum_{m=1}^{n-1} m^{p-2}\left(\sum_{\nu=m+1}^{n} \nu\left|\Delta a_{\nu}\right|\right)^{p}\right\} .
\end{array}
$$

Thus, by Lemma 5 and Lemma 6, we obtain that

$$
I_{21}^{p} \leq K\left(n^{-p} \sum_{\nu=1}^{n-1} \nu^{2 p-2} a_{\nu}^{p}+K_{2} \sum_{\nu=n}^{\infty} \nu^{p-2} a_{\nu}^{p}\right) .
$$

In a way similar to the treatment of (3.9), we can easily deduce that

$$
n^{p-1} a_{j(n+1)}^{p} \leq K n^{-p} \sum_{\nu=1}^{n-1} \nu^{2 p-2} a_{\nu}^{p}+\sum_{\nu=n}^{\infty} \nu^{p-2} a_{\nu}^{p}, \quad j=1,2,4,
$$

with applying Lemma 4, we achieve that

$$
\begin{aligned}
I_{22} & \leq\left\{\int_{\pi / 2 n}^{\pi+\pi / 2 n}\left|\sum_{\nu=n+1}^{\infty}\right| \Delta a_{\nu}|| D_{\nu}(x)||^{p} d x\right\}^{1 / p} \\
& \leq K\left|\sum_{\nu=n+1}^{\infty}\right| \Delta a_{\nu}||^{p}\left\{\int_{\pi / 2 n}^{\infty} x^{-p} d x\right\}^{1 / p} \\
& \leq K n^{1-1 / p}\left(a_{n+1}+a_{2 n+2}+a_{4 n+4}+\sum_{k=n+1}^{\infty} \frac{a_{k}}{k}\right)
\end{aligned}
$$




$$
\begin{aligned}
\leq & K n^{-1}\left(\sum_{\nu=1}^{n-1} \nu^{2 p-2} a_{\nu}^{p}\right)^{1 / p}+K\left(\sum_{\nu=n}^{\infty} \nu^{p-2} a_{\nu}^{p}\right)^{1 / p} \\
& +K\left(n^{p-1}\left(\sum_{k=n+1}^{2 n} \frac{a_{k}}{k}\right)^{p}+n^{p-1}\left(\sum_{k=2 n}^{\infty} \frac{a_{k}}{k}\right)^{p}\right)^{1 / p} \\
\leq & K n^{-1}\left(\sum_{\nu=1}^{n-1} \nu^{2 p-2} a_{\nu}^{p}\right)^{1 / p}+K\left(\sum_{\nu=n}^{\infty} \nu^{p-2} a_{\nu}^{p}\right)^{1 / p} \\
& +K\left(n^{-1}\left(\sum_{k=n}^{2 n} a_{k}\right)^{p}+\sum_{\nu=n+1}^{2 n} \nu^{p-2}\left(\sum_{k=\nu}^{\infty} \frac{a_{k}}{k}\right)^{p}\right)^{1 / p} \\
\leq & K n^{-1}\left(\sum_{\nu=1}^{n-1} \nu^{2 p-2} a_{\nu}^{p}\right)^{1 / p}+K\left(\sum_{\nu=n}^{\infty} \nu^{p-2} a_{\nu}^{p}\right)^{1 / p} \\
& +K\left(n^{p-2} \sum_{k=n}^{2 n} a_{k}^{p}+\sum_{\nu=n+1}^{\infty} \nu^{p-2}\left(\sum_{k=\nu}^{\infty} \frac{a_{k}}{k}\right)^{p}\right)^{1 / p} \\
\leq & K n^{-1}\left(\sum_{\nu=1}^{n-1} \nu^{2 p-2} a_{\nu}^{p}\right)^{1 / p}+K\left(\sum_{\nu=n}^{\infty} \nu^{p-2} a_{\nu}^{p}\right)^{1 / p} \\
\leq & K n^{-1}\left(\sum_{\nu=1}^{2 n} \nu_{k=n}^{2 p-2} a_{\nu}^{p}\right)^{1 / p}+K\left(\sum_{\nu=n}^{\infty} \nu^{p-2} a_{\nu}^{p}\right)^{1 / p} . \\
& \left.\left.+K a_{k}^{p}+\sum_{k=n}^{\infty} \frac{a_{k}}{k}\right)^{p}\right)^{1 / p} \\
\leq &
\end{aligned}
$$

Altogether, the above estimates for $I_{1}$ and $I_{2}$ complete Theorem 1.

Proof of Theorem 2. By (3.8),

$$
\sum_{k=[n / 2]}^{2 n} a_{k} \geq K n a_{n}
$$

and combining (4.2) with (3.1) of Lemma 1, we have (2.4).

By estimate (3.2) of Lemma 1, (2.7) will be proved if the following inequality

$$
\sum_{n=1}^{\infty} \lambda(n)\left(\sum_{k=n}^{\infty}\left|\Delta a_{k}\right|\right)^{r} \leq K \sum_{n=1}^{\infty} \lambda(n) a_{n}^{r}
$$

holds. Furthermore, with the help of Lemma 4, what we really need to establish is 
that

$$
\sum_{n=1}^{\infty} \lambda(n)\left(\sum_{k=n}^{\infty} \frac{a_{k}}{k}\right)^{r} \leq K \sum_{n=1}^{\infty} \lambda(n) a_{n}^{r} .
$$

In fact, if $r=1$, by exchanging the order of summation and applying (2.5), we have

$$
\begin{aligned}
\sum_{n=1}^{\infty} \lambda(n) \sum_{k=n}^{\infty} \frac{a_{k}}{k} & =\sum_{n=1}^{\infty} \frac{a_{n}}{n} \sum_{k=1}^{n} \lambda(k) k^{1 / p-1} k^{1-1 / p} \\
& \leq \sum_{n=1}^{\infty} \frac{a_{n}}{n} n^{1-1 / p} \sum_{k=1}^{n} \lambda(k) k^{1 / p-1} \leq K \sum_{n=1}^{\infty} \lambda(n) a_{n} ;
\end{aligned}
$$

if $r>1$, by Hölder's inequality and (2.5), we still have

$$
\begin{aligned}
\sum_{n=1}^{\infty} \lambda(n)\left(\sum_{k=n}^{\infty} \frac{a_{k}}{k}\right)^{r} & \leq \sum_{n=1}^{\infty} \lambda(n)\left(\sum_{k=n}^{\infty} \frac{1}{k^{1-\frac{(1-p) r}{p(r-1)}}}\right)^{r-1} \sum_{k=n}^{\infty} \frac{a_{k}^{r}}{k^{1+\frac{(1-p) r}{p}}} \\
& \leq K \sum_{k=1}^{\infty} \frac{a_{k}^{r}}{k^{1+\frac{(1-p) r}{p}}} \sum_{n=1}^{k} \lambda(n) n^{\frac{r}{p}-r} \leq \sum_{n=1}^{\infty} \lambda(n) a_{n}^{r} .
\end{aligned}
$$

Proof of Theorem 3. Most of the proof can be proceeded as the corresponding part of Leindler [7] word by word, we omit the details here.

Proof of Theorem 4. As Leindler [7] pointed out, what we need to do is to verify that $(2.8) \Rightarrow$ (i) and that (ii) $\Rightarrow(2.8)$. By applying Abel's tranformation

$$
\sum_{\nu=n}^{\infty} \lambda_{\nu} u_{\nu}=\sum_{\nu=n}^{\infty}\left(\lambda_{\nu}-\lambda_{\nu+1}\right) \sum_{k=1}^{\nu} u_{k}-\lambda_{n} \sum_{k=1}^{n-1} u_{k}
$$

with $\lambda_{\nu}=\nu^{-p}$ and $u_{\nu}=\nu^{2 p-2} a_{\nu}^{p}$, and also by (2.5), we evidently have

$$
\sum_{\nu=n}^{\infty} \nu^{p-2} a_{\nu}^{p}=\sum_{\nu=n}^{\infty} \nu^{-p} \nu^{2 p-2} a_{\nu}^{p} \leq K n^{-p},
$$

thus, the second term in (2.3) is not larger than $K n^{-1}$. Altogether, by Theorem 1, it means that $f \in \Lambda_{p}$.

Let $f(x)$ be the sum function of, say, the series $\sum_{n=1}^{\infty} a_{n} \sin n x$, and set

$$
F(x):=\int_{0}^{x} f(t) d t=\sum_{n=1}^{\infty} n^{-1} a_{n}(1-\cos n x) .
$$


An standard argument yields that

$$
F(\pi /(2 n))=2 \sum_{k=1}^{\infty} \frac{a_{k}}{k} \sin ^{2} \frac{k \pi}{4 n} \geq \frac{K}{n} \sum_{k=[n / 2]}^{2 n} a_{k},
$$

so that $F(\pi /(2 n)) \geq K a_{n}$ by $(4.2)$. Set

$$
G(x):=\int_{0}^{x} d t \int_{0}^{t}\left|f^{\prime}(u)\right| d u .
$$

Obviously, $F(x) \leq G(x)$. Hence, applying Hardy's inequality ([12]) twice, we obtain that

$$
\begin{aligned}
\sum_{n=2}^{\infty} n^{2 p-2} a_{n}^{p} & \leq \sum_{n=2}^{\infty} n^{2 p-2} G^{p}(\pi /(2 n)) \leq K \sum_{n=2}^{\infty} n^{2 p-2} G^{p}(\pi / n) \\
& \leq K \sum_{n=2}^{\infty} \int_{\pi / n}^{\pi /(n-1)}\left[\frac{G(x)}{x}\right]^{p} x^{-p} d x \leq K \int_{0}^{\pi}\left[\frac{G(x)}{x}\right]^{p} x^{-p} d x \\
& \leq K \int_{0}^{\pi}\left(\int_{0}^{x}\left|f^{\prime}(t)\right| d t\right)^{p} x^{-p} d x \leq K \int_{0}^{\pi}\left|f^{\prime}(x)\right|^{p} d x<\infty .
\end{aligned}
$$

Proof of Theorem 5. Set $T_{m, 2 n}(x):=\sum_{\nu=m}^{2 n} \cos \nu x$, then $([1]$ or $[7])$

$$
I_{m, 2 n, t}:=\int_{-\pi}^{\pi}(2 f(x)-f(x+t)-f(x-t)) T_{m, 2 n}(x) d x=4 \pi \sum_{\nu=m}^{2 n} a_{\nu} \sin ^{2} \frac{1}{2} \nu t .
$$

Taking $t=\pi / n$ and $m=[n / 2]$ in (4.3), then applying (4.2) again, we have

$$
\sum_{\nu=m}^{2 n} a_{\nu} \sin ^{2} \frac{1}{2} \nu t \geq K \sum_{\nu=m}^{2 n} a_{\nu} \geq n a_{n}
$$

On the other hand, we have

$$
\int_{-\pi}^{\pi}\left|T_{m, 2 n}(x)\right|^{q} d x \leq K\left\{\int_{0}^{\pi /(2 n)} n^{q} d x+\int_{\pi /(2 n)}^{\pi} x^{-q} d x\right\} \leq K n^{q-1}
$$

By Hölder's inequality, it follows that

$$
I_{m, 2 n, \pi / n} \leq K n^{1 / p}\left\{\int_{-\pi}^{\pi}\left|f\left(x+\frac{\pi}{n}\right)+f\left(x-\frac{\pi}{n}\right)-2 f(x)\right|^{p} d x\right\}^{1 / p} \leq K n^{1 / p} \omega_{p}^{*}(f, \pi / n) .
$$

A combination of (4.3) and (4.4) leads to

$$
\omega_{p}^{*}(f, 1 / n) \geq K n^{1-1 / p} a_{n}
$$


Therefore, from that $\omega_{p}^{*}(f, 1 / n) \leq K n^{-1}$ and by (4.5), we get $a_{n} \leq K n^{-2+1 / p}$, whence by Theorem 1 , it follows that

$$
\omega_{p}(f, 1 / n) \leq K n^{-1}(\log n)^{1 / p},
$$

and $(2.9)$ is done.

\section{References}

1. S. Aljančić, On the integral moduli of continuity in $L^{p}(1<p<\infty)$ of Fourier series with monotone coefficients, Proc. Amer. Math. Soc., 17(1966), 287-294.

2. R. J. Le and S. P. Zhou, A new condition for the uniform convergence of certain trigonometric series, Acta Math. Hungar., 108(2005), 161-169.

3. L. Leindler, Generalization of inequalities of Hardy and Littlewood, Acta Sci. Math. (Szeged), 31(1970), 279-285.

4. L. Leindler, On cosine series with positive coefficients, Acta Math. Hungar., 22(1971), 397-406.

5. L. Leindler, On the uniform convergence and boundedness of a certain class of sine series, Anal. Math., 27(2001), 279-285.

6. L. Leindler, A new class of numerical sequences and its applications to sine and cosine series, Anal. Math., 28(2002), 279-286.

7. L. Leindler, Relations among Fourier series and sum-functions, Acta Math. Hungar., 104(2004), 171-183.

8. D. S. Yu and S. P. Zhou, A generalization of monotonicity and applications, submitted to Acta Math. Hungar..

9. D. S. Yu and S. P. Zhou, On belonging of trigonometric series to Ba spaces, to appear. 
10. S. P. Zhou and R. J. Le, A remark on "two-sided" monotonicity condition: an application to $L^{p}$ convergence, Acta Math. Hungar. accepted.

11. S. P. Zhou and R. J. Le, A new condition for the uniform convergence in Fourier analysis, Adv. Math. (Beijing), 33(2004), 567-569.

12. A. Zygmund, Trigonometric Series, 2nd. Ed., Vol.I, Cambridge Univ. Press, Cambridge, 1959.

\section{Yu Dansheng}

Institute of Mathematics

Zhejiang Sci-Tech University

Xiasha Economic Development Area

Hangzhou Zhejiang 310018 China

e-mail: danshengyu@yahoo.com.cn

\section{Zhou Songping}

Institute of Mathematics

Zhejiang Sci-Tech University

Xiasha Economic Development Area

Hangzhou Zhejiang 310018 China

e-mail: szhou@zjip.com 